\title{
Cerebral Blood Flow in Children with Spontaneous Occlusion of the Circle of Willis (Moyamoya Disease): Comparison with Healthy Children and Evaluation of Annual Changes
}

\author{
Satoshi Kuroda, Hiroyasu KamiYama, Hiroshi ABE, Toru YamaUCHI, \\ Yoshihiko KoHAMA, Kiyohiro HoukIN and Kenji MitsumORI* \\ Department of Neurosurgery, Hokkaido University School of Medicine, Sapporo; \\ ${ }^{*}$ Hokkaido Neurosurgical Memorial Hospital, Sapporo
}

\begin{abstract}
Changes in the mean cerebral blood flow (mCBF) with growth in normal children (1-20 years old) were evaluated, and compared to serial $\mathrm{mCBF}$ changes in children with spontaneous occlusion of the circle of Willis (moyamoya disease) after vascular reconstructive surgery. $\mathrm{mCBF}$ gradually decreased with growth in control children. Preoperative $\mathrm{mCBF}$ was similar or decreased in moyamoya disease patients with transient ischemic attack, and slightly decreased in those with cerebral infarction. Postoperative mCBF gradually decreased similarly to physiological changes with growth in controls. These results suggest that changes with age should be considered when preoperative and long-term cerebral hemodynamics are evaluated in children with moyamoya disease.
\end{abstract}

Key words: circle of Willis, moyamoya disease, cerebral blood flow, growth, vascular reconstruction

\section{Introduction}

Most children with spontaneous occlusion of the circle of Willis (moyamoya disease) present with initial symptoms of cerebral ischemia. We previously evaluated the efficacy of surgical vascular reconstruction for childhood moyamoya disease using cerebral angiography, measurements of cerebral hemodynamics with single photon emission computed tomography (SPECT), and electroencephalography. The best results were obtained with superficial temporal artery-middle cerebral artery (STA-MCA) anastomosis combined with indirect vascular reconstruction extending to the frontal region. ${ }^{1-3,8,18)}$

However, the cerebral hemodynamics in children change with growth. ${ }^{1-13,16)} \mathrm{We}$ found that the postoperative cerebral blood flow (CBF) in childhood moyamoya disease decreased gradually with age although the cerebral perfusion reserve was maintained. ${ }^{3)}$ This study evaluated the changes in $\mathrm{CBF}$

Received September 2, 1992; Accepted December 2, 1992 with growth in normal children, and in the pre- and postoperative $\mathrm{CBF}$ in children with moyamoya disease compared with the CBF in age-matched normal controls.

\section{Materials and Methods}

The study included 26 children with moyamoya disease (25 definitely diagnosed and 1 suspected) treated at our department during the past 10 years. The age at onset was 2-10 years old. Sixteen patients developed transient ischemic attack (TIA) and the other 10 cerebral infarction. All patients underwent surgical vascular reconstruction: indirect vascular reconstruction in 17 hemispheres [encephalo-duroarterio-synangiosis (EDAS) in 3, encephalo-myosynangiosis in 5, and encephalo-myo-arterio-synangiosis (EMAS) in 9], and combined with STA-MCA anastomosis in 34 hemispheres [EMAS in 7 and encephalo-duro-arterio-myo-synangiosis (EDAMS) in 27]. The follow-up period was 6 months to 10 years (mean 4.9 yrs).

The control measurements were made in 67 
hemispheres in 39 children (1-20 years old) with underlying diseases having a negligible effect on CBF, including epilepsy unaccompanied by organic lesions in eight children, minor head injuries (bruise, cerebral concussion, linear skull fracture, and depressed skull fracture) in 13, meningitis in three, small cerebral infarction in the basal ganglia in five (only contralateral hemispheres were examined in the chronic stage), and others in 10.

$\mathrm{CBF}$ was measured using the ${ }^{133} \mathrm{Xe}$ inhalation method and SPECT (HEADTOME SET-031; Shimadzu Co., Kyoto). The absolute values of regional $\mathrm{CBF}$ (rCBF) were measured using the sequential picture method. ${ }^{\text {" The spatial resolution was }}$ $20 \mathrm{~mm}$ in the plane and $24 \mathrm{~mm}$ axially, measured as full width at half maximum. The data obtained from two slices, centered at 50 and $85 \mathrm{~mm}$ above and parallel to the orbitomeatal line, were used as the mean $\mathrm{CBF}(\mathrm{mCBF})$ in the entire hemisphere.

\section{Results}

The $\mathrm{mCBF}$ in the 67 normal hemispheres gradually decreased with growth, showing a negative correlation $(r=-0.6778, p=0.01)$ (Fig. 1).

The preoperative $\mathrm{mCBF}$ in 11 children with moyamoya disease was compared with age-matched control values (Fig. 2). Most children who developed TIA had a mCBF similar to the controls, although some showed a lower value. In contrast, the mCBF in the children with cerebral infarction was slightly lower than in the controls.

Annual changes were evaluated in 17 patients dur-

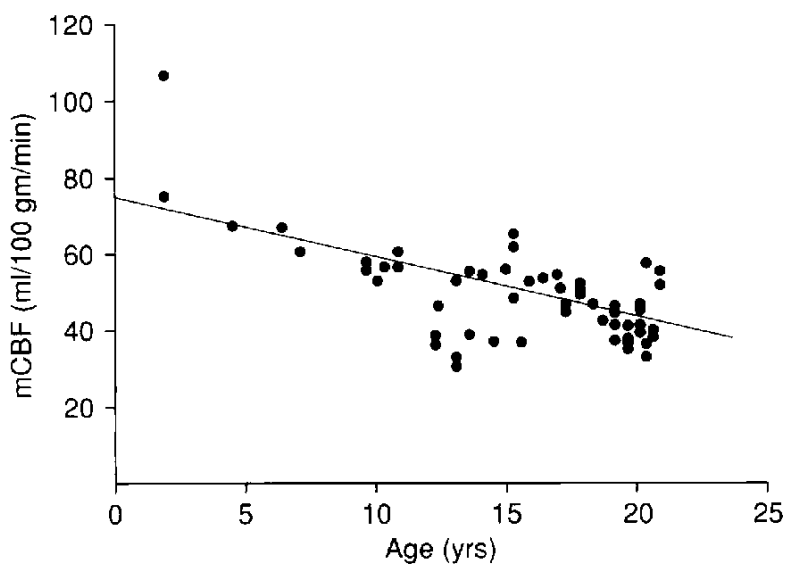

Fig. 1 Relationship between age and $\mathrm{mCBF}$ in both hemispheres in control group, showing negative correlation $(\mathrm{Y}=-1.766 \mathrm{X}+75.24, \mathrm{r}=$ -0.6778 ).

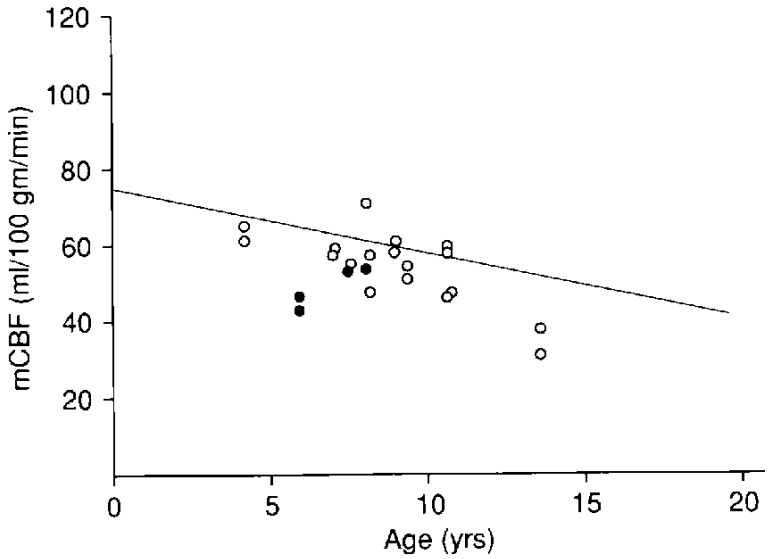

Fig. 2 Relationship between age and preoperative $\mathrm{mCBF}$ in both hemispheres in 11 children with moyamoya disease. $O$ : patients with TIA, - : patients with infarction. Regression line is for control group.
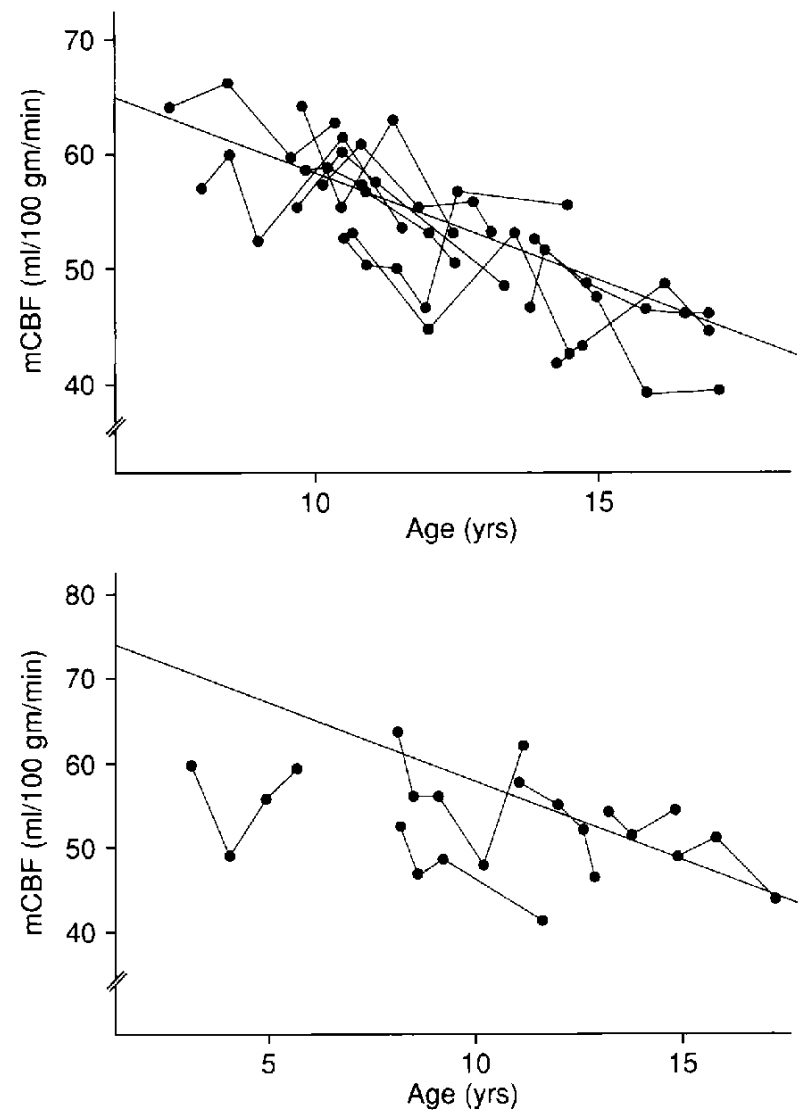

Fig. 3 Serial changes of postoperative mCBF in unilateral hemisphere in the 11 children who developed TIA (upper) and in the six children who developed completed stroke (lower). Regression line is for the control group. 


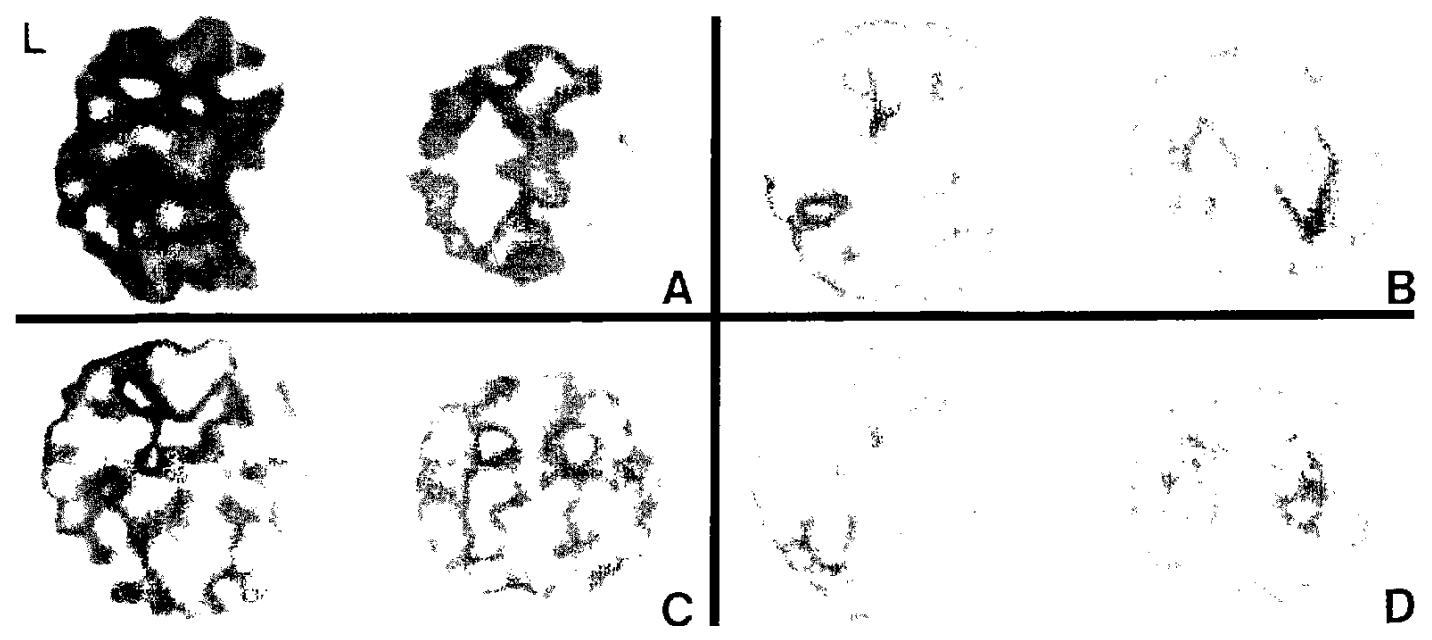

Fig. $4{ }^{13 .} \mathrm{Xe}$ SPECT scans before and after bilateral ST A-MCA anastomoses and EDAMS in Case 1. A: Preoperative CBF study at age 13.5 years, revealing marked hypoperfusion especially in the right MCA. B: Postoperative CBF study 6 months after operation, showing normal mCBF and normal rCBF distribution. C, D: Postoperative CBF studies 2.5 (C) and 3.5 years (D) after operation, showing a gradual decrease in mCBF. He has experienced no ischemic episode since the operation.

$\mathrm{L}$
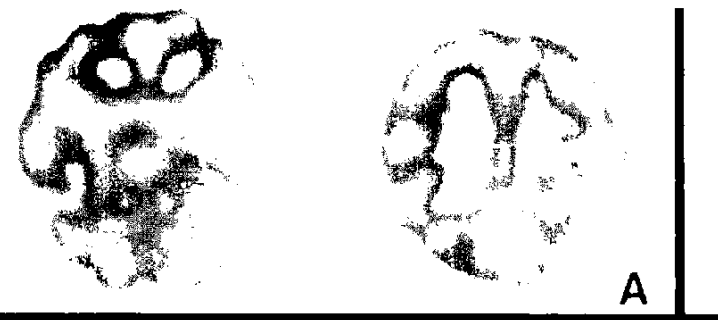

B
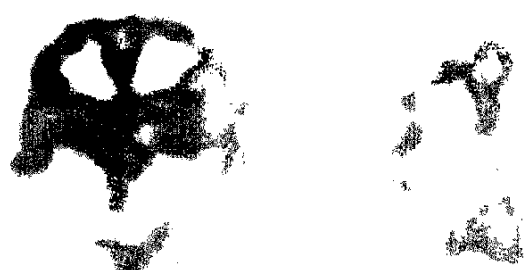

?
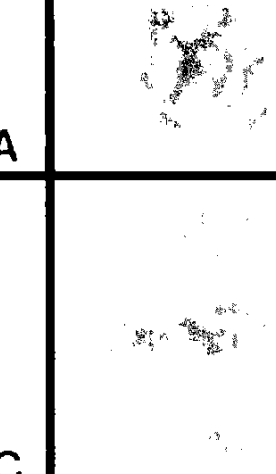

C

D

Fig. $5 \quad{ }^{13 .} \mathrm{Xe}$ SPECT scans after bilateral STA-MCA anastomoses and EDAMS in Case 2. No preoperative CBF study was performed. A, B: CBF studies at age 14 (A) and 15 years (B), revealing normal $\mathrm{mCBF}$ and normal rCBF distribution. C, D: Follow-up CBF studies at age $16(\mathrm{C})$ and 17 years (D), showing a gradual decrease in mCBF. Normal $\mathrm{rCBF}$ was maintained and she has experienced no ischemic attack.

ing follow-up periods of 2 years or more after vascular reconstruction. The 11 children with TIA showed a gradual decrease in MCBF with growth similar to that in the controls. The six children with cerebral infarction also showed a similar trend, although the number of the patients was few (Fig. 3).
No patients demonstrating a gradual decrease in $\mathrm{mCBF}$ experienced any episode of cerebral ischemia after operation.

Illustrative cases are presented.

Case 1: A 13-year-old male frequently suffered TIA, and was admitted. CT scans revealed no abnormal- 
ities. Moyamoya disease was diagnosed based on cerebral angiograms. He underwent bilateral STAMCA anastomoses and EDAMS at age 13.5 years. Immediately after operation, the attacks of cerebral ischemia ceased, and no neurological abnormalities were observed. $C B F$, which preoperatively decreased in the entire brain especially in the right MCA territory, became normal after operation. Follow-up studies showed a gradual decrease in $\mathrm{CBF}$ during the 3.5 years after operation (Fig. 4).

Case 2: A 11-year-old female experienced TIA, and was admitted. Moyamoya disease was diagnosed based on cerebral angiograms. She underwent bilateral STA-MCA anastomoses and EDAMS at age 11 years. Postoperatively, no ischemic attack has occurred. No preoperative CBF studies were performed. Serial CBF measurements demonstrated a gradual decrease in $\mathrm{mCBF}$ with normal pattern of rCBF during the 6-year follow-up period (Fig. 5).

\section{Discussion}

Previous studies on cerebral hemodynamics in children show that CBF is low in neonates but much higher in children than in adults. ${ }^{11-13,16)}$ Kennedy and Sokoloff ${ }^{(1)}$ demonstrated that the average global CBF in nine normal children (age 3-11 yrs) was about 1.8 times that of normal young adults. Ogawa et al. ${ }^{16)}$ found that CBF in children less than 5 years old was about twice that in adults, decreases rapidly with growth until approximately 10 years old, and then gradually thereafter until adulthood.

Cerebral metabolism, which is closely associated with cerebral circulation, may also undergo certain changes with growth. ${ }^{4,7,11,127}$ Kennedy and Sokoloff ${ }^{11}$ showed that average cerebral oxygen utilization was approximately 1.3 times higher in children than in adults. Chugani et al. ${ }^{4}$ measured local cerebral metabolic rates for glucose (ICMRGlc) in 29 children (age 5 days-15.1 yrs), using 2-deoxy-2 $\left[{ }^{18}\right.$ F ffluoro-Dglucose and positron emission tomography (PET), concluding that absolute values of ICMRGlc were low at birth, rose by age 3-4 years, and continued at high levels until about 9 years old, when they began to decrease, reaching adult values by the latter part of the second decade. Such serial changes in CBF and metabolism are considered to be closely associated with the neuron density in the cerebral cortex. ${ }^{7,12)}$

In this study, CBF correlated negatively with age in controls, consistent with previous findings. ${ }^{11-13,16)}$ However, since there were only a few controls less than 5 years old, further studies are needed in this age range.
Many investigators have measured the CBF in children with moyamoya disease, using two- or three-dimensional methods such as the ${ }^{133} \mathrm{Xe}$ inhalation method, stable xenon CT, SPECT, and PET ${ }^{6,10,14,15,17,18)}$ Karasawa et al. ${ }^{1(0)}$ evaluated rCBF in 67 child and 18 adult patients using stable xenon $\mathrm{CT}$, showing that cerebral ischemia occurs in the borderzone area between the MCA and anterior cerebral artery, but not in the basal ganglia. Kuyama et al. ${ }^{14)}$ reported that $\mathrm{mCBF}$ was reduced in the ipsilateral hemispheres of two children with completed stroke, but there was no significant difference with bilateral $\mathrm{mCBF}$ in six children with TIA. Gotoh et al. ${ }^{6}$ reported the disappearance of hyperfrontality in adult patients. Taki et al ${ }^{(7)}$ measured $\mathrm{rCBF}$, regional oxygen extraction fraction ( $\mathrm{rOEF}$ ), regional cerebral metabolic rate for oxygen, and regional cerebral blood volume (rCBV) in five children using ${ }^{15} \mathrm{O}_{2}$ steady-state methods and PET, finding a mild decrease in perfusion pressure, dilated resistant vessels, and prolonged circulation time, because of decreased $\mathrm{rCBF} / \mathrm{rCBV}$ ratio and preserved rOEF.

However, few studies on cerebral hemodynamics provided comparisons with healthy children, who show dramatic changes in cerebral circulation and metabolism with growth. Ogawa et al. ${ }^{15}$ investigated hemispheric $\mathrm{CBF}$ in moyamoya disease patients using intravenous ${ }^{133} \mathrm{Xe}$ injection methods, reporting that CBF was lower than in normal children, especially in the frontal lobe.

This study compared preoperative $\mathrm{mCBF}$ in moyamoya disease patients with age-matched controls. The $\mathrm{mCBF}$ was normal or decreased in patients with TIA, and slightly decreased in those with cerebral infarction. Further detailed evaluation is required for $\mathrm{mCBF}$ and $\mathrm{rCBF}$, since regional ischemia is often present in the internal carotid artery territory centering on the frontal lobe in children with moyamoya disease.

Studies of hemodynamic changes after vascular reconstruction have demonstrated improved $\mathrm{rCBF}$ in the affected cortical regions after indirect reconstruction such as EDAS, EMAS, and EDAMS. ${ }^{5,14)}$ Takikawa et al. ${ }^{18}$ also showed that STA-MCA anastomosis combined with indirect reconstruction could improve $\mathrm{rCBF}$ in more extensive regions, especially the frontal lobe.

No long-term follow-up of postoperative cerebral hemodynamics in children with moyamoya disease have been made. In this study, a gradual decrease in CBF was observed in the patients followed for more than 2 years after vascular reconstruction. These changes were similar to those in controls. Since no patient has developed cerebral ischemic attacks and 
mCBF was maintained within normal limits, we conclude that these changes are associated with physiological changes in cerebral circulation with growth, not deterioration of cerebral hemodynamics caused by moyamoya disease. Such physiological changes should be taken into consideration in longterm follow-up after vascular reconstruction. Our patients will be carefully followed up to clarify whether postoperative $\mathrm{CBF}$ changes until adulthood are similar to those in healthy children, and whether cerebral ischemic or hemorrhagic attacks develop in adulthood.

\section{References}

1) Abe H, Kamiyama H: Surgical treatment of the occlusion of the circle of Willis, in: Annual Report 1986 of the Research Committee on Spontaneous Occlusion of the Circle of Willis of the Ministry of Health and Welfare Japan. 1987, pp 37-42 (in Japanese)

2) Abe H, Kamiyama H, Takikawa S, Kuroda S: Rebuild up finding on EEG in child moyamoya disease: Correlation with Diamox test on ${ }^{133} \mathrm{Xe}$ SPECT, in: Annual Report 1988 of the Research Committee on Spontaneous Occlusion of the Circle of Willis of the Ministry of Health and Welfare Japan. 1989, pp 103110 (in Japanese)

3) Abe H, Kamiyama $H$, Takikawa $S$, Kuroda $S$, Yamauchi T: Intellectual development of children with moyamoya disease, in: Annual Report 1989 of the Research Committee on Spontaneous Occlusion of the Circle of Willis of the Ministry of Health and Welfare Japan. 1990, pp 126-132 (in Japanese)

4) Chugani HT, Phelps ME, Mazziotta JC: Positron emission tomography study of human brain functional development. Ann Neurol 22: 487-497, 1987

5) Fukui M, Matsushima $T$, Kuwabara W, Ichiya $Y$, Wada M, Hasuo K: Hemodynamic evaluation of moyamoya disease in children by positron emission computed tomography before and after neurosurgical procedures (EDAS, EMS, EMAS), in: Annual Report 1986 of the Research Committee on Spontaneous Occlusion of the Circle of Willis of the Ministry of Health and Welfare Japan. 1987, pp 9198 (in Japanese)

6) Gotoh F, Fukuuchi Y, Takashima S, Kawamura J, Terayama Y: Local cerebral blood flow and $\mathrm{CO}_{2}$ responsiveness in patients with "moyamoya disease," in: Annual Report 1986 of the Research Committee on Spontaneous Occlusion of the Circle of Willis of the Ministry of Health and Welfare Japan. 1987, pp 78-87 (in Japanese)

7) Hatazawa J, Brooks RA, Chiro GD, Bachrach SL: Glucose utilization rate versus brain size in human. Neurology 37: 587-588, 1987

8) Isobe M, Kuroda S, Kamiyama H, Abe H, Mitumori
$\mathrm{K}$ : Cerebral blood flow reactivity to hyperventilation in children with spontaneous occlusion of the circle of Willis (moyamoya disease). No Shinkei Geka 20: 399-407, 1992 (in Japanese)

9) Kanno 1, Lassen NA: Two methods for calculating cerebral blood flow from emission tomography of inert gas concentration. J Comput Assist Tomogr 3: 71-76, 1979

10) Karasawa J, Kikuchi H, Miyamoto S: CT findings of "moyamoya" disease: Correlation with the cerebral blood flow. CT Kenkyu 6: 373-379, 1984 (in Japanese)

11) Kennedy C, Sokoloff L: An adaptation of the nitrous oxide method to the study of the cerebral circulation in children: Normal values for cerebral blood flow and cerebral metabolic rate in childhood. J Clin Invest 36 : $1130-1137,1957$

12) Kety SS: Human cerebral blood flow and oxygen consumption as related to aging. $J$ Chronic Dis 3: 478486,1956

13) Komatani A, Yamaguchi K, Kera M: Cerebral blood flow and brain size in healthy subjects: Sensitivity to age and gender. No To Shinkei 42: 889-893, 1990 (in Japanese)

14) Kuyama $H$, Niimi $H$, Kawauchi $M$, Tanimoto $T$, Murota T, Kinugasa K, Asari S, Nishimoto A, Nagao $S:$ Hemodynamic changes after vascular reconstructive surgery for moyamoya disease. CT Kenkyu 10: 317-322, 1988 (in Japanese)

15) Ogawa A, Nakamura N, Sakurai Y, Kayama T, Wada T, Suzuki J: Cerebral blood flow in moyamoya disease. No To Shinkei 39: 199-203, 1987 (in Japanese)

16) Ogawa A, Nakamura N, Sugita K, Sakurai $Y$, Kayama T, Suzuki J: Regional cerebral blood flow in children: Normal values and regional distribution of cerebral blood flow in childhood. No To Shinkei 39: 113-118, 1987 (in Japanese)

17) Taki W, Yonekawa $Y$, Kobayashi A, Ishikawa $M$, Kikuchi H, Nishizawa S, Senda M, Yonekura Y, Fukuyama H, Harada K, Kameyama M: Cerebral circulation and oxygen metabolism in children's "moyamoya" diseases. No Shinkei Geka 16: 557562, 1988 (in Japanese)

18) Takikawa S, Kamiyama $\mathrm{H}$, Abe $\mathrm{H}$, Mitsumori $\mathrm{K}$, Tsuru M: Hemodynamic evaluation of vascular reconstructive surgery for childhood moyamoya disease using single photon emission computed tomography. Neurol Med Chir (Tokyo) 30: 389-395, 1990

Address reprint requests to: S. Kuroda, M.D., Department of Neurosurgery, Hokkaido University School of Medicine, North-15, West-7, Kita-ku, Sapporo 060, Japan. 